\title{
Regulating Internal Electric Field by Breaking Lattice Symmetry in Polymeric Photocatalysts for Visible-Light- Driven Disinfection
}

Dawei Zhang,+[a,b] Xiangrong Li,+[a,c] Yukun Yan, ${ }^{*}[a]$ Zihao Rei Gao,[d] Hanwen Wang,[a] Jinsong Zhang, ${ }^{*}[a]$ Jun Xiao*[a]

[a] D. Zhang, Dr. X. Li, Dr. J. Xiao, Y. Yan, H. Wang, Prof. J. Zhang

Shenyang National Laboratory for Materials Science, Institute of Metal Research, Chinese Academy of Sciences Shenyang, 110016, China

E-mail: jxiao14b@imr.ac.cn; ykyan@imr.ac.cn; jszhang@imr.ac.cn

[b] D. Zhang

School of Materials Science and Engineering, University of Science and Technology of China Shenyang 110016, Liaoning, China

[c] Dr. X. Li

College of light industry, Liaoning University 66 Chongshan Middle Road, Shenyang 110036, China

[d] Z. R. Gao

Instituto de Ciencia de Materiales de Madrid, Consejo Superior de Investigaciones Científicas (ICMM-CSIC)

c/ Sor Juana Inés de la Cruz, 3, Madrid, 28049, Spain

[+] Those authors contributed equally to this work.

Abstract:The inefficient charge separation and transport (CST) process has been a major obstacle that limits the catalytic activity of polymeric photocatalysts. Here we propose a symmetry-breaking strategy to regulate the internal electric field (IEF) in crystalline polymers for enhanced CST and photocatalytic efficiency. As a proof of concept, two crystalline linear copolymers, $p$-phenylenediamine $/ m$-phthalaldehyde (PDA-MAH) and $p$ phenylenediamine/ $p$-phthalaldehyde (PDA-PAH), were synthesized. By breaking the lattice symmetry, we observed robust IEF in the non-centrosymmetric PDA-MAH, as compared to the centrosymmetric PDA-PAH. Such strong IEF was extended throughout the catalyst bulk to accelerate the CST process in PDA-MAH, resulting in a photocatalytic disinfection performance under visible light irradiation that surpassed the best of the previously reported polymeric photocatalysts. This work presents a promising paradigm for tailoring polymeric semiconductors with efficient CST process.

Utilizing sunlight as a renewable energy source, photocatalytic technology is a promising approach to addressing a number of the energy and environmental problems that the world faces today.$^{[1]}$ Recently, polymeric photocatalysts have attracted much attention because of their fascinating properties, such as tunable electronic structures, environment benignity and low-cost processability, etc. ${ }^{[2]}$ However, the poor charge separation and transport (CST) process has undermined photocatalytic efficiency in most polymeric photocatalysts. ${ }^{[3]}$ One 
way to gain an additional driving force for the CST process in polymers is the introduction of donor-acceptor (D-A) moieties. ${ }^{[4]}$ Once properly designed, the D-A moieties with dipole moments can generate the local internal electric field (IEF), ${ }^{[5]}$ which facilitates the separation of photogenerated charges via intramolecular charge transfer. ${ }^{[6]}$ However, building D-A moieties with high dipole moments often requires elaborate design and complex synthesis,,$^{[7]}$ thus limiting the large-scale application of this approach. Moreover, the highly dipolar D-A moieties tend to remain in anti-parallel arrangement, ${ }^{[8]}$ which is unfavourable for the generation of distinct dipole moments in the polymer bulk,,$^{[9]}$ resulting in weak CST process throughout the material. Therefore, it's highly desired to find a suitable strategy that can increase the dipole moments and, hence, strengthen the IEF over the long range throughout whole bulk, in order to improve the efficiency of the CST process and the related photocatalytic performance in polymeric photocatalysts. ${ }^{[10]}$

Inspired by the lattice distortion strategy, which was shown to be highly effective in enhancing IEF in the bulk $\mathrm{TiO}_{2}$ and $\mathrm{BiPO}_{4}$ system, ${ }^{[11]}$ the present study applied a similar lattice symmetry-breaking strategy on the typical crystalline conjugated polymers, poly(phenylene-imine) (PPI). These facile-to-prepare PPIs from the intermediates of fine chemicals (phenylenediamine and phthalaldehyde) possess identical functional groups, weak D-A moieties, and quite different lattice symmetries. As shown in Scheme 1, when the lattice symmetry was broken deliberately, the elimination of symmetric centre would introduce non-overlap positive and negative charge centres, creating dipole moments in the lattice. Therefore, a remarkable IEF would be built up along the dipole direction throughout the whole bulk. ${ }^{[12]}$ In this work, we verified the feasibility of the hypothesis above by demonstrating that PDA-MAH, which had crystallized in monoclinic structure without lattice symmetry centre, yielded a much stronger IEF than the orthorhombic PDA-PAH and the amorphous MDA-MAH. The robust IEF of PDA-MAH greatly accelerated the CST process, leading to a rapid visible-light-driven water disinfection with 7 logs (>99.9999\%) inactivation of $E$. coli within $1 \mathrm{~h}$, which is the highest among those with previously reported polymeric photocatalysts.

The PPIs were synthesized via acetic acid catalyzed Schiff base reaction in the mixed solvents (see Supporting Information). $p$-/m-phenylenediamine (PDA/MDA) and $p-/ m$ phthalaldehyde (PAH/MAH) were selected as the monomers to build the PPIs, and three copolymers were referred to as PDA-PAH, PDA-MAH, and MDA-MAH, respectively (Figure S1). As shown in the Fourier transform infrared (FTIR) spectra (Figure S2), the identical bands of the three PPIs samples at $1640 \mathrm{~cm}^{-1}$ clearly demonstrated that the imine $(C=N)$ bonds had formed by the Schiff-base condensation. The formation of $\mathrm{C}=\mathrm{N}$ bonds was further confirmed by X-ray photoelectron spectroscopy (XPS) as N1s peaked at around $398.8 \mathrm{eV}$ in all the spectra (Figure S3 and S4). The resonances during $100-160 \mathrm{ppm}$ in ${ }^{13} \mathrm{C}$ cross-polarization (CP) magic-angle spinning (MAS) NMR spectra corresponded to the $C$ atoms on the benzene rings from the phthalaldehyde and phenylenediamine moieties (Figure S5), confirming the substitution relationships on the benzene ring of the respective three PPIs. Noting that the resonances at around $150 \mathrm{ppm}$ were attributed to the $\mathrm{C}$ atoms in the imine groups.

To distinguish the bulk phase structures, three PPIs were characterized by powder X-ray diffraction (PXRD). Both PDA-PAH and PDA-MAH were highly crystalline while MDA-MAH 
was amorphous (Figure S6). Le Bail fitting of the PXRD patterns of PDA-PAH and PDA$\mathrm{MAH}$, shown in Figure $1 \mathrm{a}$ and $1 \mathrm{~b}$, indicated that PDA-PAH had an orthorhombic structure with unit cell parameters of $a=22.915 \AA, b=10.746 \AA, c=7.578 \AA$, (space group: $P m c m$ ) and PDA-MAH assumed a monoclinic structure with lattice parameters of $a=10.666 \AA, b$ $=10.912 \AA, c=7.051 \AA$, and $\beta=96.200^{\circ}$ (space group: Pm). The symmetry breaking at the lattice level from the orthorhombic to the monoclinic structure eliminates the symmetric center in the unit cell, hence, creating dipole moments in the lattice. Apparently, the symmetry reduction of the monomers may have led to the lattice symmetry breaking of the bulk phase crystals. Under the transmission electron microscope (TEM), the crystallized PPIs (i.e. PDA-MAH and PDA-PAH) adopted typical 2D nanosheet morphology (Figure 1c, $1 \mathrm{~d}$ ); the atomic force microscopy showed $\sim 4.5 \mathrm{~nm}$ thickness for the same two PPIs nanosheets (Figure S7). Hence, two crystallized PPIs have similar chemical structure and morphology, and their primary difference lies at the centrosymmetry due to symmetry breaking at the lattice level.

To investigate the buildup of IEF following the symmetry breaking, the surface charge density and the Zeta potential were measured in three PPI samples by the Kelvin probe force microscope (KPFM) and from the electrophoretic mobility, as both were recently used as the descriptors of the IEF. ${ }^{[13]}$ As shown in Figure 2a (S8), PDA-MAH exhibited the highest surface potential $(\Delta \mathrm{E}=205 \mathrm{mV})$ among three PPIs, five times than that of PDA$\mathrm{PAH}(40 \mathrm{mV})$. The Zeta potentials of three PPIs followed the same trend as the KPFM surface potential (Figure 2b), with the highest Zeta potential found in PDA-MAH. Thus, we can conclude that the PDA-MAH had the most robust IEF. Since PDA-MAH and PDA-PAH both were well crystallized, the difference in the IEF of those two samples would be mainly caused by the symmetry reduction in crystal structures, as their oligomer dipoles were similar (Figure S9). Since the irregular chain arrangement counteracted the monomer dipole, the amorphous MDA-MAH demonstrated remarkably weaker IEF. The much stronger IEF in PDA-MAH is expected to add significant driving force in the CST process for the photocatalyst.

Aside from the CST process, photocatalytic activity also depends on the photon absorption process. However, ultraviolet diffuse reflectance spectra (UV-DRS, Figure 2c) showed the close absorbance stopping edges of three PPIs prepared here, indicating similar light absorption at visible wavelengths. The appreciable visible light harvest ability and appropriate band structures (Figure S10, concluded from UV-DRS spectra, XPS valence band spectra in Figure S11, and Mott-Schottky curves in Figure S12-S13) pointed to the possible photocatalytic capability of the PPIs thermodynamically, while the major differences in the kinetic process, especially the CST process, were observed as presented below.

First, photoluminescence spectra (Figure S14) demonstrated that PDA-MAH had the lowest recombination of photoexcited electron-hole pairs. Second, transient fluorescence spectroscopy revealed that the transient fluorescence lifetime of PDA-MAH was longer than those of the other two PPIs, as an indication that the robust IEF in PAD-MAH had enhanced the separation and migration rate of the photogenerated charge carriers (Figure 2d). Thirdly, electrochemical impedance spectroscopy (EIS) showed that PDA-MAH had a much smaller semicircular Nyquist plot radius than other PPIs, especially under light 
illumination (Figure 2e), suggesting a lower charge transfer resistance in PDA-MAH bulk. Fourthly, the strong transient photocurrent response of PDA-MAH provided further evidence that the robust IEF of PDA-MAH accelerated the CST process in the bulk (Figure S15), which could boost photocatalytic performance in the kinetic aspect. The photocatalytic activities of the as-synthesized PPI samples were then evaluated by their photocatalytic disinfection performance under visible light illumination. Using $E$. coli as the target indicators, the kinetics of the photocatalytic antibacterial activity of PPI samples were examined in terms of the bacterial survival ratio as a function of the photocatalytic treatment time. As shown in Figure 3a, PDA-MAH under visible light exhibited over $99.99999 \%$. coli inactivation in just 1 hour, whereas PDA-PAH acted very slowly at a rate of about $99 \%$ disinfection efficiency after $120 \mathrm{~min}$; and the MDA-MAH showed negligible toxic effect on $E$. coli over the same time period. For reference, comparison experiments demonstrated that visible light irradiation alone caused no photolysis in E. coli and all of the PPI samples had no toxic effect on bacterial cells without the light illumination. Obviously, the strong IEF in PDA-MAH had boosted photocatalytic activity vastly. More strikingly, we found that PDAMAH exhibited the best catalytic bacterial disinfection rate under visible light irradiation as compared with those of reported metal-free photocatalysts (Figure S16 and Table S1), indicating its great potential in future applications in water treatment practice.

Given the superior disinfection performance of PDA-MAH, the photocatalytic mechanism was further studied. Reactive species (RS) quenching experiments demonstrated that $\mathrm{H}_{2} \mathrm{O}_{2}$ generated in situ during the photocatalytic process played the dominant role in bacterial inactivation among all the RSs (Figure 3b) since the addition of the $\mathrm{H}_{2} \mathrm{O}_{2}$ scavengers significantly reduced the photocatalytic disinfection rate. When the concentration of in situ formed $\mathrm{H}_{2} \mathrm{O}_{2}$ was measured over time, the $\mathrm{H}_{2} \mathrm{O}_{2}$ concentration initially increased with light exposure time, then became relatively stable (ca. $6 \mu \mathrm{M})$ with prolonged illumination time (Figure S17), which is congruent with the process where the $\mathrm{H}_{2} \mathrm{O}_{2}$ is continuously produced and consumed dynamically. Furthermore, rotating disk electrode (RDE) studies revealed that the $\mathrm{H}_{2} \mathrm{O}_{2}$ on PDA-MAH was generated through the two-electron oxygen reduction pathway (Figure S18, S19). To gain more insight into the $2 \mathrm{e}^{-}$oxygen reduction pathway at the molecular level, density functional theory (DFT) calculations were performed to determine the thermodynamic energy for the critical step in $\mathrm{H}_{2} \mathrm{O}_{2}$ production. Since recent studies have identified that the $\mathrm{O}_{2}$ adsorption and generation of endoperoxide species is the critical step to reduce $\mathrm{O}_{2}$ into $\mathrm{H}_{2} \mathrm{O}_{2}$ on polymeric photocatalyst, ${ }^{[14]}$ we compared the energies of formation of $\mathrm{O}_{2}{ }^{*}$ on the four different active sites. The calculations showed that the endoperoxide species had favorable Gibbs free energies when anilino or imine $(\mathrm{C}=\mathrm{N})$ moieties acted as the $\mathrm{O}_{2}$ adsorption sites (Figure S20). The calculations further confirmed both the energies of formation of $\mathrm{O}_{2}{ }^{*}$ and $\mathrm{OOH}^{*}$ on the imine site were much smaller than that on aniline, suggesting that imines tend to be the active site of the two-electron reduction pathway (Figure 3c). Based on the above analysis, the selective $\mathrm{H}_{2} \mathrm{O}_{2}$ formation mechanism on the photoactive PDA-MAH is depicted in Figure $\mathrm{S} 21$, where the molecular $\mathrm{O}_{2}$ was first spontaneously adsorbed on the imine site to produce endoperoxide species, and the endoperoxide species then reacted with $2 \mathrm{e}^{-}$and $\mathrm{H}^{+}$to form $\mathrm{OOH}^{*}$ species. Subsequent protonation of the $\mathrm{OOH}^{*}$ species finally produced $\mathrm{H}_{2} \mathrm{O}_{2}$ and completed the photocatalytic cycle. 
Encouraged by the above findings, we further explored the application potential of PDAMAH for disinfection in air decontamination using the face mask as a model. By coating the PDA-MAH photocatalysts on the middle layer of the face mask, the antibacterial performance of the PDA-MAH coated mask was compared with that of a commercial mask (N95). As a proof of concept, a semi-closed system reactor was built in which a mask was used to divide the reactor chamber into two separate parts (Figure S22). ${ }^{[15]}$ After flowing bioaerosols from the inlet, through the mask, and over the remaining reactor chamber, the exhaust air stream was exposed to the bacterial collectors at the exit of the reactor, and the number of bacteria surviving on the collector plates was counted. As shown in Figure $3 \mathrm{~d}$, PDA-MAH mask showed $>99 \%$ E. coli inactivation in 60 min under visible light irradiation, which is greatly superior to the commercial mask (N95) under visible light $(<96 \%)$ and PDA-MAH mask in dark $(<94 \%)$ over the same time period. Helium ion microscope provided more intuitive evidence for the demonstration experiment above (Figure S23). Residual bacteria attached to the PDA-MAH mask were observed after the dark reaction experiment but seldom found after the visible light irradiation with photocatalysis. In addition, repeated experiments showed that the PDA-MAH mask still maintained sufficient activity after four runs, suggesting the high stability of PDA-MAH for air sterilization (Figure S24). The remarkable bactericidal activity thus attested to the great protective coating function of PDA-MAH.

In conclusion, we proposed and validated a new strategy for accelerating the CST process of polymeric bulk photocatalysts through breaking lattice symmetry in highly crystallized PPI nanosheets. By using this strategy, a highly efficient photocatalyst PDA-MAH was synthesized facilely from the fine chemicals intermediates $p$-phenylenediamine and $m$ phthalaldehyde. PDA-MAH showed strong internal electric field, fast CST process, and a prominent photocatalytic disinfection performance under visible lights irradiation that surpassed the best of the previously reported polymeric photocatalysts. Importantly, this work highlights the significance of lattice symmetry control in polymeric photocatalysts for regulating the CST process and boosting the photocatalytic activity, and provides a new way for polymer semiconductor designs besides modifying polymer chemical structures.

[1] a) Q. Wang, K. Domen, Chem. Rev. 2020, 120, 919-985; b) C. Liu, D. Kong, P.-C. Hsu, H. Yuan, H.-W. Lee, Y. Liu, H. Wang, S. Wang, K. Yan, D. Lin, P. A. Maraccini, K. M. Parker, A. B. Boehm, Y. Cui, Nat. Nanotechnol. 2016, 11, 1098-1104.

[2] a) T. Banerjee, F. Podjaski, J. Kröger, B. P. Biswal, B. V. Lotsch, Nat. Rev. Mater. 2021, 6, 168-190; b) C. Dai, Y. Pan, B. Liu, Adv. Energy Mater. 2020, 10, 2002474; c) Z. Teng, N. Yang, H. Lv, S. Wang, M. Hu, C. Wang, D. Wang, G. Wang, Chem 2019, 5, 664-680.

[3] H. Wang, S. Jin, X. Zhang, Y. Xie, Angew. Chem. Int. Ed. 2020, 59, 22828-22839; Angew. Chem. 2020, 132, $23024-23035$.

[4] a) Z.-A. Lan, M. Wu, Z. Fang, X. Chi, X. Chen, Y. Zhang, X. Wang, Angew. Chem. Int. Ed. 2021, 60, 16355-16359; Angew. Chem. 2021, 133, 16491 -16495; b) H. Ou, X. Chen, L. Lin, Y. Fang, X. Wang, Angew. Chem. Int. Ed. 2018, 57, 8729-8733; Angew. Chem. 2018, 130, 8865 -8869; c) C. Shu, C. Han, X. Yang, C. Zhang, Y. Chen, S. Ren, F. Wang, F. Huang, J.-X. Jiang, Adv. Mater. 2021, 33, 2008498; d) Z.-A. Lan, Y. Fang, Y. Zhang, X. Wang, Angew. Chem. Int. Ed. 2018, 57, 470-474; Angew. Chem. 2018, 130, 479 -483; e) 
C. Yang, B. C. Ma, L. Zhang, S. Lin, S. Ghasimi, K. Landfester, K. A. I. Zhang, X. Wang, Angew. Chem. Int. Ed. 2016, 55, 9202-9206; Angew. Chem. 2016, 128, 9348 -9352.

[5] a) Q. Xie, Y. Yang, W. Zhang, Z. Gao, X. Li, J. Tang, C. Pan, G. Yu, Chem. Sci. 2021, 12, 5631-5637; b) Z. Zhang, X. Chen, H. Zhang, W. Liu, W. Zhu, Y. Zhu, Adv. Mater. 2020, 32, 1907746.

[6] M. Krzeszewski, E. M. Espinoza, C. Červinka, J. B. Derr, J. A. Clark, D. Borchardt, G. J. O. Beran, D. T. Gryko, V. I. Vullev, Angew. Chem. Int. Ed. 2018, 57, 12365-12369; Angew. Chem. 2018, 130, $12545-12549$.

[7] a) K. Müllen, W. Pisula, J. Am. Chem. Soc. 2015, 137, 9503-9505; b) C. B. Kc, G. N. Lim, F. D'Souza, Angew. Chem. Int. Ed. 2015, 54, 5088-5092; Angew. Chem. 2015, 127, $5177-5181$.

[8] a) J. Zyss, J. Chem. Phys. 1993, 98, 6583-6599; b) T. Marszalek, M. Li, W. Pisula, Chem. Commun. 2016, 52, 10938-10947.

[9] a) J. Xu, C. Yang, S. Bi, W. Wang, Y. He, D. Wu, Q. Liang, X. Wang, F. Zhang, Angew. Chem. Int. Ed. 2020, 59, 23845-23853; Angew. Chem. 2020, 132, 24053 - 24061; b) J. Zyss, I. Ledoux, Chem. Rev. 1994, 94, 77-105.

[10] F. Chen, H. Huang, L. Guo, Y. Zhang, T. Ma, Angew. Chem. Int. Ed. 2019, 58, 1006110073; Angew. Chem. 2019, 131, 10164 - 10176.

[11] a) Y. Hu, Y. Pan, Z. Wang, T. Lin, Y. Gao, B. Luo, H. Hu, F. Fan, G. Liu, L. Wang, Nat. Commun. 2020, 11, 2129; b) C. Pan, D. Li, X. Ma, Y. Chen, Y. Zhu, Catal. Sci. Technol. 2011, 1, 1399-1405.

[12] a) Y. Guo, W. Shi, Y. Zhu, EcoMat 2019, 1, e12007; b) L. Li, P. A. Salvador, G. S. Rohrer, Nanoscale 2014, 6, 24-42.

[13] a) J. Yang, J. Jing, Y. Zhu, Adv. Mater. 2021, 33, 2101026; b) J. Jing, J. Yang, Z. Zhang, Y. Zhu, Adv. Energy Mater. 2021, 11, 2101392.

[14] a) L. Chen, L. Wang, Y. Wan, Y. Zhang, Z. Qi, X. Wu, H. Xu, Adv. Mater. 2020, 32, 1904433; b) Y. Zhao, P. Zhang, Z. Yang, L. Li, J. Gao, S. Chen, T. Xie, C. Diao, S. Xi, B. Xiao, C. Hu, W. Choi, Nat. Commun. 2021, 12, 3701.

[15] P. Li, J. Li, X. Feng, J. Li, Y. Hao, J. Zhang, H. Wang, A. Yin, J. Zhou, X. Ma, B. Wang, Nat. Commun. 2019, 10, 2177.

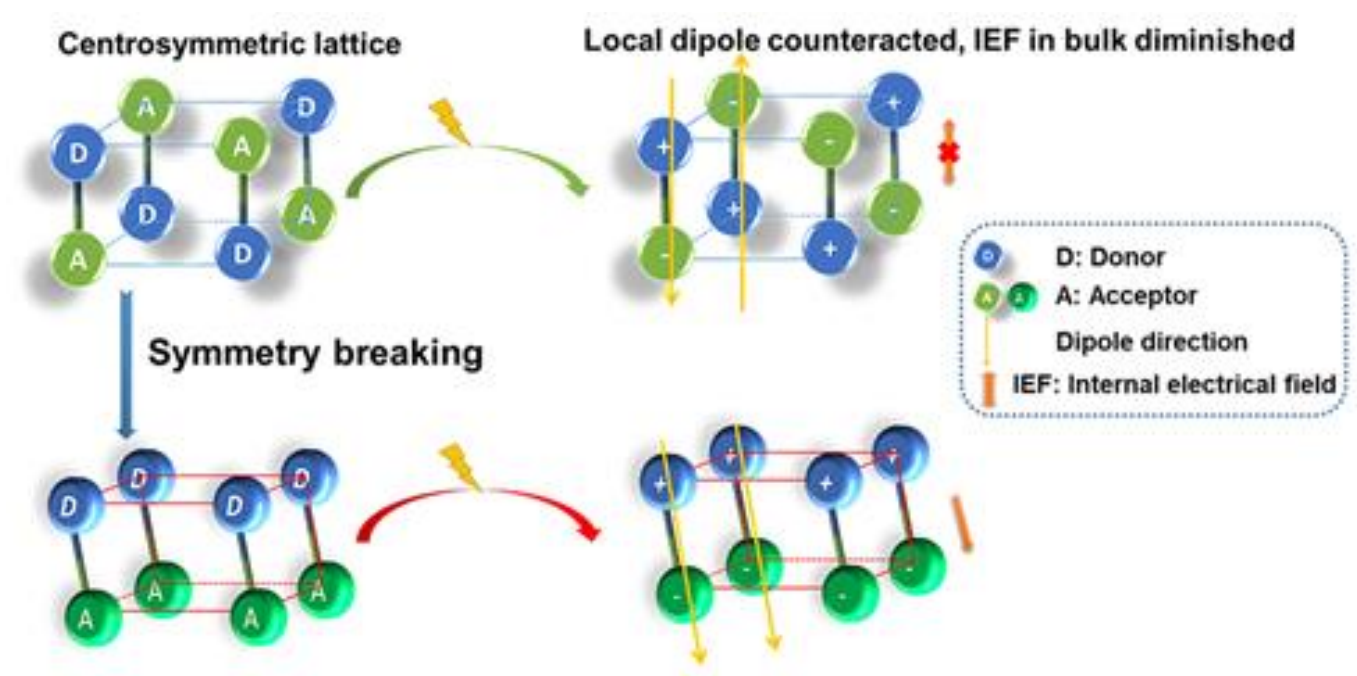

Dipole moments in unit cell, accumulated into IEF in bulk 
Scheme 1. Illustration of lattice symmetry-breaking enhance internal electrical field in highly crystalline conjugated polymers with weak D-A moieties, phenylenediamine as weak donor, and phthalaldehyde as weak acceptor.
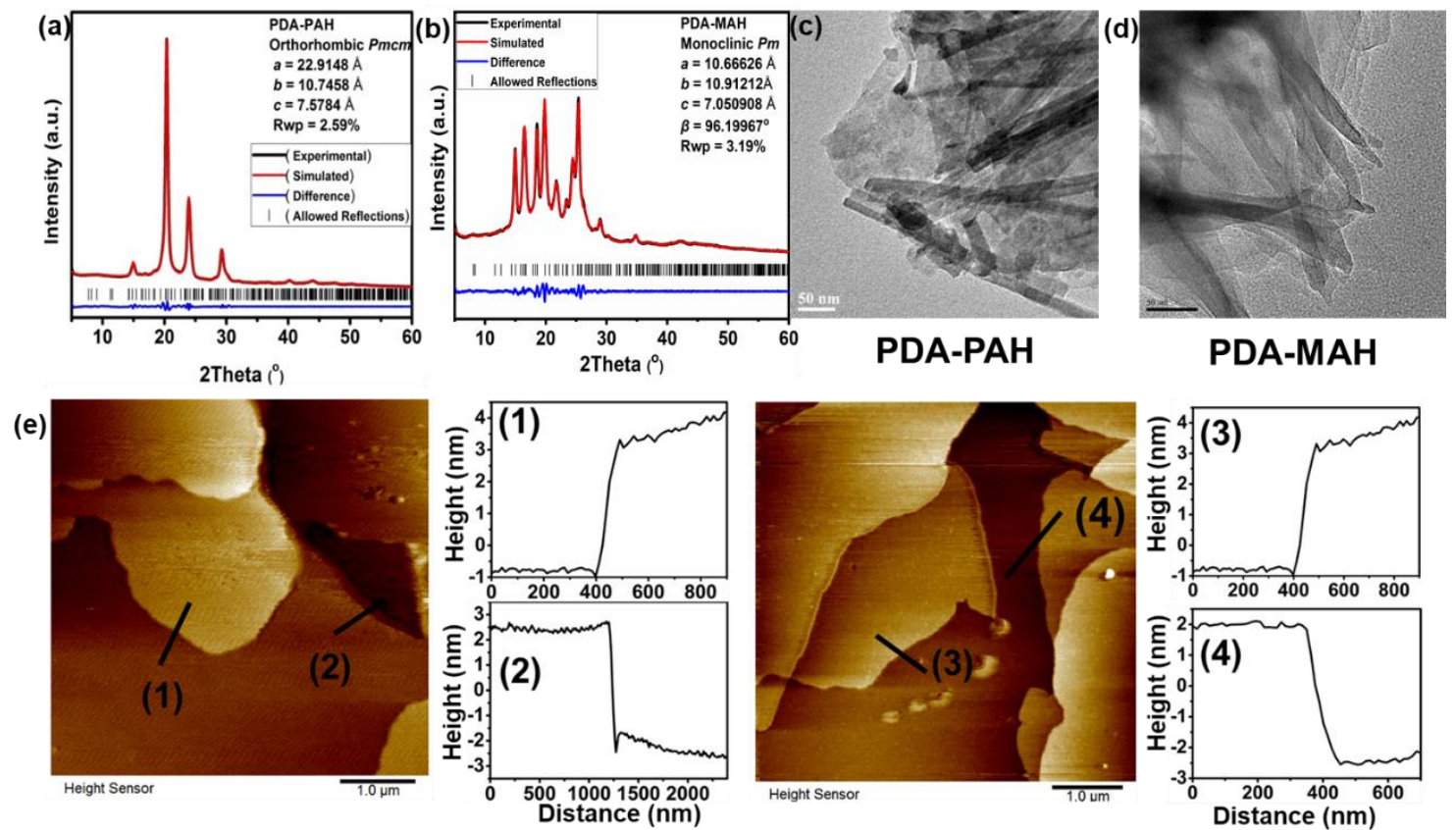

Figure1 PXRD pattern and Le Bail fitting plots of (a) PAH-PDA and (b) PDA-MAH;TEM images of PDA- PAH(c) and PDA-MAH(d); Liner scanning of thickness of PDA-PAH(e) and PDA-MAH(f) by AFM 

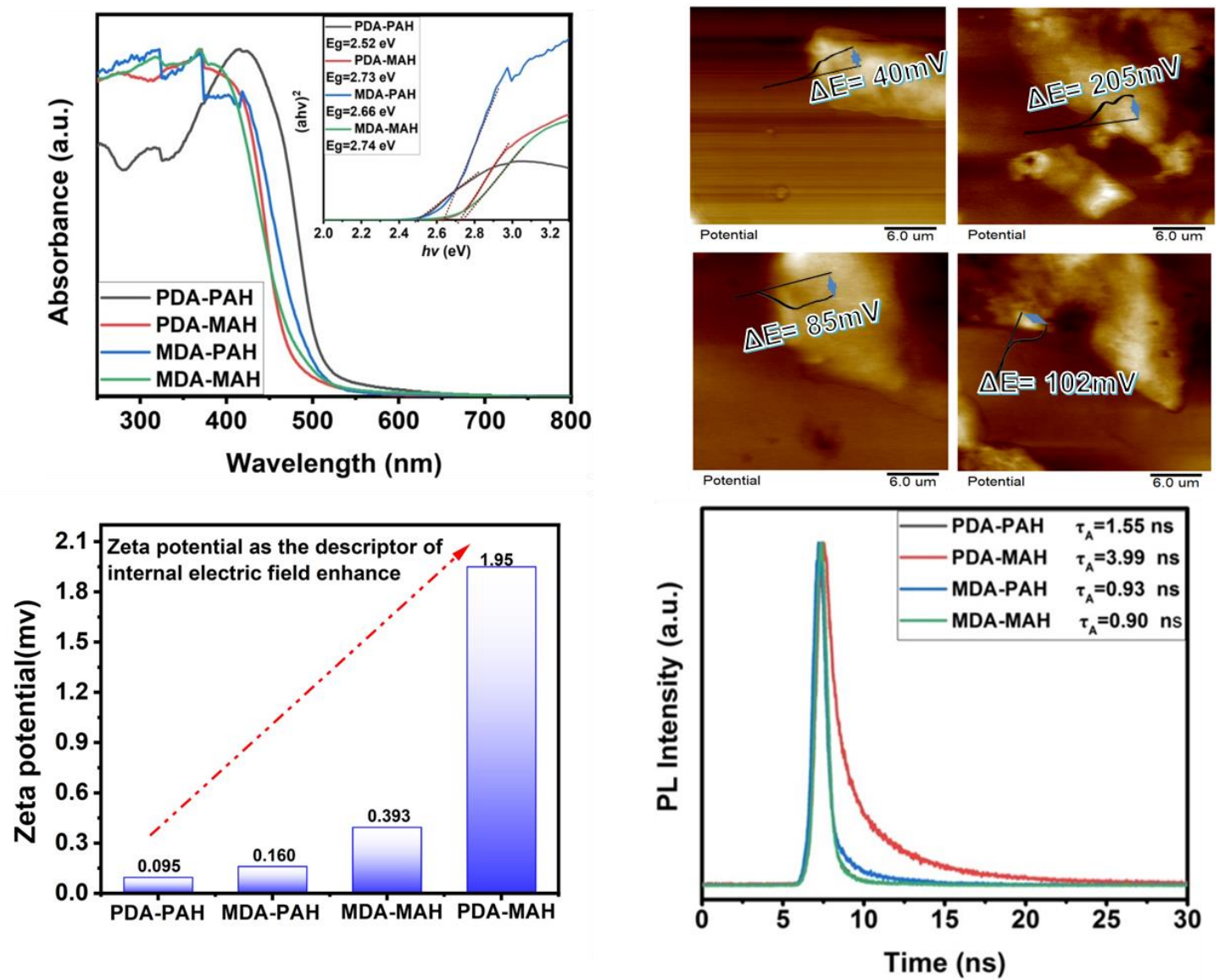

Figure2 The thermodynamic basis and kinetic factors (internal electric field) for PPI photocatalysts a)UV-vis-NIR diffuse reflection Spectra and (inset) corresponding band gap energies of PPIsb)Surface potential of four PPIs etected with KPFM. c)Zeta potential of PPIs. d) Transient fluorescence spectrum of the PPIs.
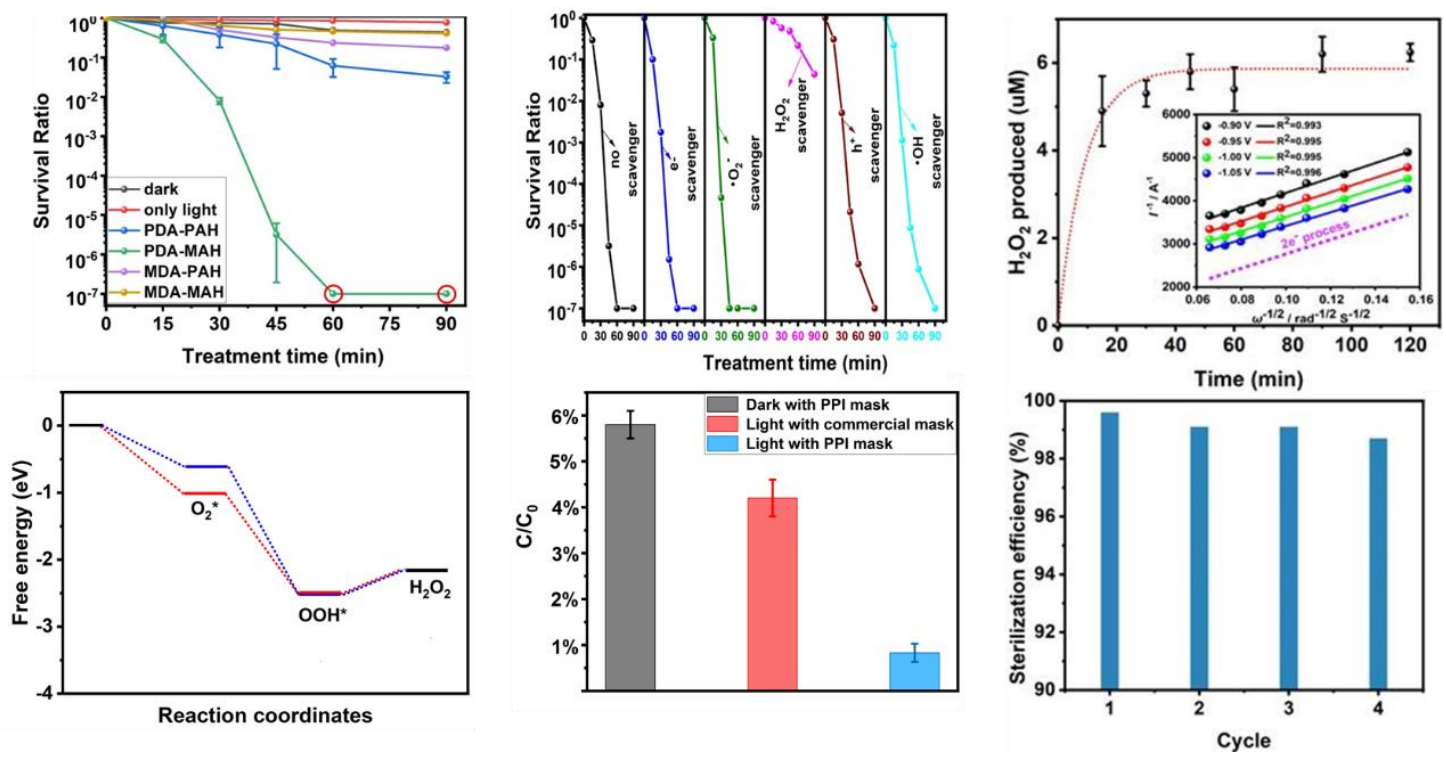

Figure 3 Visible light driven disinfection performance and mechanism of PPIs a)Visible light driven disinfection curves of PPIs with control experiments; b) Photocatalytic disinfection performance 
of PDA-MAH under different scavenger conditions; c) The $\mathrm{H} 2 \mathrm{O} 2$ concentrations in PDA-MAH test solution and Koutecky-Levich (K-L) plots of PDA-MAH(inset); d) Free energy diagrams of $\mathrm{H} 2 \mathrm{O} 2$ generation on different active site of PDA-MAH; e) Comparison of the air disinfection performance between PDA-MAH coating mask and commercial mask f) cycling air disinfection test of PDA-MAH coating mask 